\title{
A Novel Octagonal UWB Antenna for Cognitive Radio
}

\author{
S.Siva sundara pandian ${ }^{1}$, Dr.C.D.Suriyakala ${ }^{2}$ \\ Sathyabama University Chennai-600 119, India
}

\begin{abstract}
This paper presents about a new antenna design of ultra wide band antenna for cognitive radio wireless communication. . This antenna consists of an irregular octagon (polygon with 8 sides) aperture on a printed circuit board; ground plane. This antenna was simulated in ADS (Advanced Design System) Momentum. . The feeding point is the micro strip line and to this attached the octagon structure. For an antenna to be suitable for channel sensing it should possess an Omni directional radiation pattern. The octagon micro strip patch antenna is the sensing antenna which detects the spectrum and provides the available range $(35-45 \mathrm{GHz})$ for the antenna to communicate. The resonant frequency of the antenna is $40 \mathrm{GHz}$.
\end{abstract}

Keywords: cognitive radio; micro-strip patch; polygon patch; sensing antenna;

\section{Introduction}

Today there is no freely available spectrum for future generation. Around $2.5 \mathrm{GHz}$ the spectrum is completely occupied. So by using the cognitive radio the spectrum can be efficiently utilized. A Cognitive Radio is a radio that can easily identify freely available spectrum and autonomously change its transmission parameters based on the interaction with the complex environment (radio scene, application and user requirements) in which it operates. The spectrum can be efficiently utilized by using the cognitive radio. Since the cognitive radio is always looking for freely available spectrum it needs a wide band receiver and wide band antenna for spectrum sensing. In the cognitive radio with the help of wide band antenna the spectrum can be sensed because it is always looking for freely available spectrum over a broader bandwidth. A wide band antenna with omni directional radiation pattern is necessary in order to achieve the channel sensing. A Cognitive Radio is a radio which can automatically change its parameters based on the environment in which it operates. The licensed users that are primary users use the spectrum only for $10 \%$ of time. The rest of the time the spectrum is idle. So by using the cognitive radio the secondary users that are unlicensed users can use the spectrum without interfering with the primary users. So the Cognitive Radio should have the ability of a wide band spectrum sensing over a broader band width since it is always looking for freely available spectrum.

In [1\} A new reconfigurable antenna is designed for Cognitive Radio. It consists of two structures incorporated together into the same substrate. In [2] a high broad side gain MEMS based reconfigurable antenna was designed for four frequency bands. A new family of microstrip-fed ultrawideband (UWB) antennas with multiple (triple/quadruple) notched bands is proposed. The notched bands are generated by a band notched filter composed of double stepped impedance resonators (SIRs)[3]. A polarization reconfigurable antenna capable of providing any arbitrary polarization by just switching the feed phasing unit is discussed in [4]. Cognitive radios may temporarily use the spectrum as long as they do not interfere with the primary users that own the license to that spectrum [5-7]. This paper reviews the possible antenna configurations for cognitive radio like wideband antennas, polarization and frequency reconfigurable antennas [8]. In [9] a wideband circular monopole and a narrowband dipole antenna for cognitive radio were presented with impedance bandwidth of the wideband monopole antenna is from $4.25-10 \mathrm{GHz}$ and narrowband dipole antenna's central frequency is $5.8 \mathrm{GHz}$. A single-port small-size antenna based on an ultra-wideband (UWB) design and has a reconfigurable band pass filter integrated in its feed line for cognitive radio (CR) applications is presented in [10]. In [11] wideband, multi-band or reconfigurable antennas have been developed and when considering the interference levels at the receiver, reconfigurable antennas are the best option, since only one single band is used at a given time. A Vivaldi wideband antenna is a good prospect for wide band sensing. This paper [12] presents two different antennas which have been developed to address the specific demands of portable cognitive radio handsets. Each antenna combines UWB and narrowband functionality within a small space. [13] A compact, CPW-fed octagon shape aperture antenna for lower band UWB applications is presented. This antenna consists 
of a irregular octagon (polygon with 8 sides) aperture on a printed circuit board, ground plane and a octagon-shaped exciting stub. Initially the proposed antenna is designed with rectangular aperture and then the rectangular shaped aperture is converted into irregular octagon shaped aperture by inserting an isosceles right angled triangular metallic shape in the corners of rectangular aperture to increase the impedance matching in the required bandwidth. The octagon shaped stub and polygon aperture (slot) are simple and has less parameter, which are convenient to optimize. The proposed antenna is designed on an inexpensive FR4-epoxy substrate. [14] The higher order regular polygonal loop antenna generally possesses higher directivity but poorer broadband impedance property. As the operating frequency becomes higher its directivity increases remarkably and the broadband property becomes more prominent. To achieve the desired properties one may select one of the many design data sets with a trade-off between broadband property and directivity.

In this paper a new ultra wideband tri-band antenna is designed for cognitive radio wireless communication. Section two describes about the geometry and dimensions of the proposed antenna. In section three we explained about results and discussion. Section four explains about conclusion

\section{Geometry Of Proposed Antenna}

In the cognitive radio we need to sense the spectrum and communicate through it. A wide band antenna is necessary in order to be able to achieve the channel sensing. The structure is shown in the figure 1. The top layer is the slotted polygon shaped patch. The bottom layer is the partial ground. It is fed via a micro strip line to produce radiation above and below the substrate. The chosen substrate is FR4. All the simulations are done using the ADS momentum. The corresponding antenna return loss is shown in the fig.3. Fig. 1 shows our proposed antenna. It consists of irregular octagonal shaped microstrip antenna in the ground of a dielectric substrate with a size of $50 \times 90 \mathrm{mil}$, a permittivity of $\varepsilon$, thickness of $h$ and loss tangent of $\tan \delta$. Fig. 1, shows the simulated irregular octagonal Microstrip antenna. The slot is excited by an open ended $60 \Omega$ micro strip transmission line of length $\mathrm{L}$. This line is connected to a $50 \Omega$ line with length tap. Good matching can be achieved by changing the length L. Specifications of proposed antennas on a substrate (FR-4) with $\mathrm{h}=1.6 \mathrm{~mm}$, $\varepsilon r=4.4$ and $\tan \delta=0.0018$. All dimensions are in $\mathrm{mm}$ except $\alpha$ which is indicated in degrees. In order to provide evidence for the antenna effectiveness design, many parameters were measured. The efficiency of the matching device is proved by the minimum return loss S11, which influences the remnant characteristics such as Directivity, Gain and band width. The microstrip line is used as the feed for the antenna and above that is the irregular octagonal aperture antenna which provides the ultra wide band coverage. The irregular octagonal aperture antenna is the sensing antenna which detects the spectrum and provides the available range for the antenna to communicate. The shape of antenna is highly designed to cover the wide band and provide spectrum reuse compatibility. The reuse of the

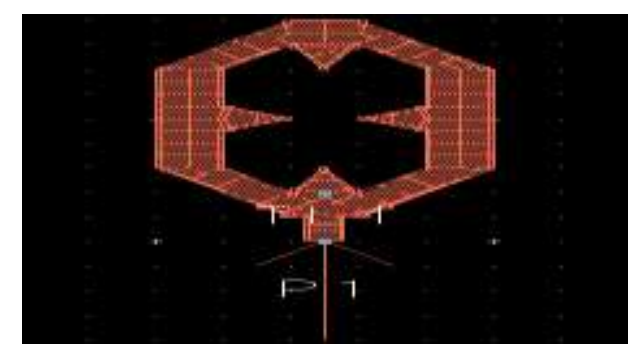

Fig.1 Simulated irregular octagonal aperture antenna

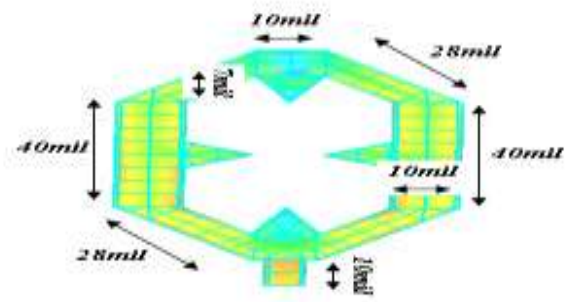

Fig.2, The dimensions of proposed antenna 


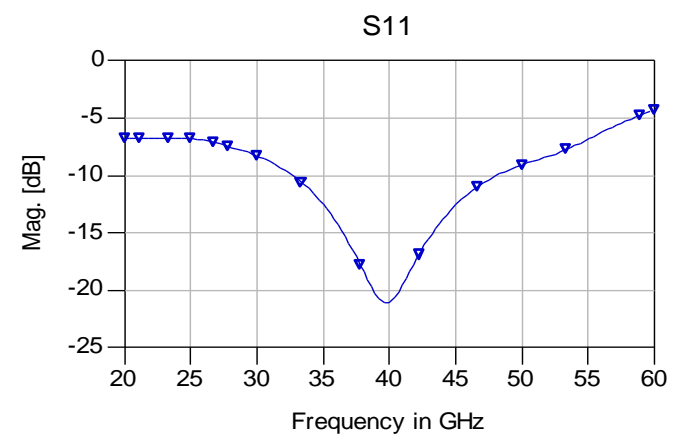

Fig.3 Return loss

spectrum is highly intelligent and highly efficiency. Fig. 2 shows the dimensions of proposed antenna. In this antenna opposite sides are equal as shown in figure 2.The dimensions are $40 \mathrm{mil}, 28 \mathrm{mil}$ and 10 mil for the respective sides. The width of the antenna is $10 \mathrm{mil}$ and 7 mil respectively. The inside projections are used to get wideband width.

\section{Results And Discussion}

Validation of our analysis are demonstrated and discussed in this section defined as follows. Simulating the effectiveness and extracting the antenna parameters and plotting currents. Simulating a conventional irregular octagonal microstrip patch antenna and comparing results. Fig.3 shows the return loss for the proposed antenna which is less than $-20 \mathrm{~dB}$ at $40 \mathrm{GHz}$.Fig.4 shows the Input impedance for irregular octagonal microstrip patch antenna Fig.5describes the phase difference in irregular octagonal microstrip patch antenna. Fig. 6 shows the simulated result for radiation pattern of E theta. For which magnitude is equal to 1.967 and phase is -25.6 degrees Fig.7 shows the simulated result for radiation pattern of $\mathrm{E}$ phi. For which magnitude is equal to 0.0002 and phase is 7.17 degrees. Fig. 8 Simulated result for radiation pattern of angle of Umax. For which theta is equal to 27 degrees and phase is 90 degrees..Fig. 9 shows the simulated results for linear polarization. Fig.10 shows the simulated results for circular polarization. Fig.11 shows the simulated results for efficiency which is equal to $50 \%$. Fig. 12 shows the simulated results for directivity and gain. The directivity is a measure of the directional properties of the antenna compared to those of an isotropic antenna. The radiation efficiency of the antenna defined as the ratio of the radiated power Pr to the input power Pi ( $0<\mathrm{er}<1)$. Validation of our analysis are demonstrated and discussed in this section defined as follows: Evaluating the accuracy of the matching impedance antenna resulting from simulating the return loss S11. The radiation pattern and the antenna parameters for the frequency of $40 \mathrm{GHz}$ is provided in the fig. 8. The antenna parameters are summarized in table.1.

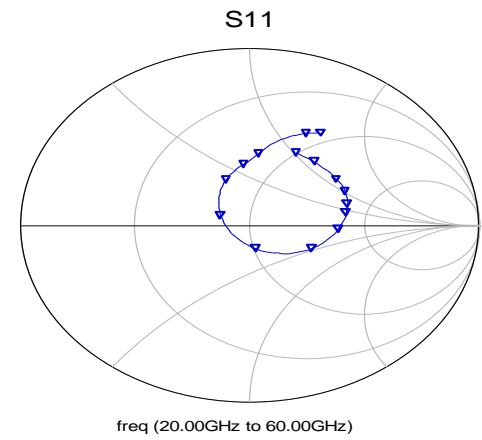

Fig.4 Input impedance for octagon antenna 


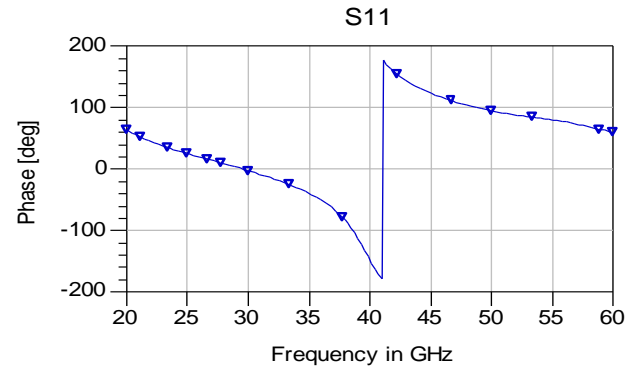

Fig. 5 phase difference in octagon antenna

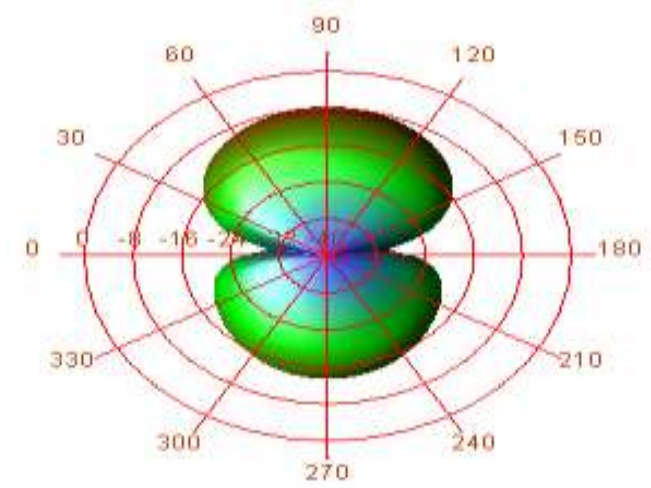

Fig.6 Simulated result for radiation pattern of E theta

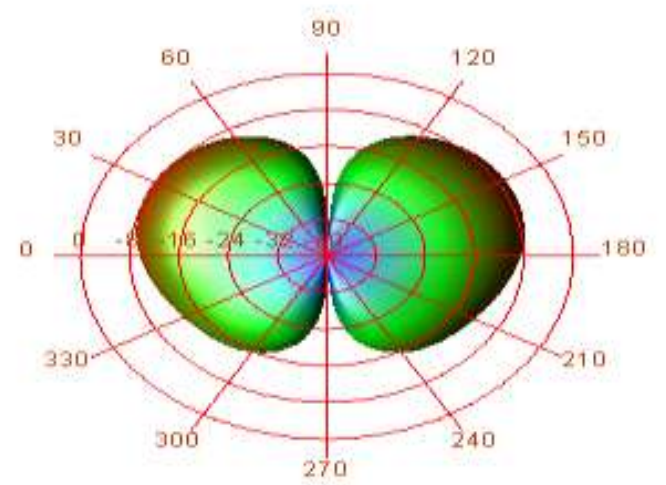

Fig.7 Simulated result for radiation pattern of E phi

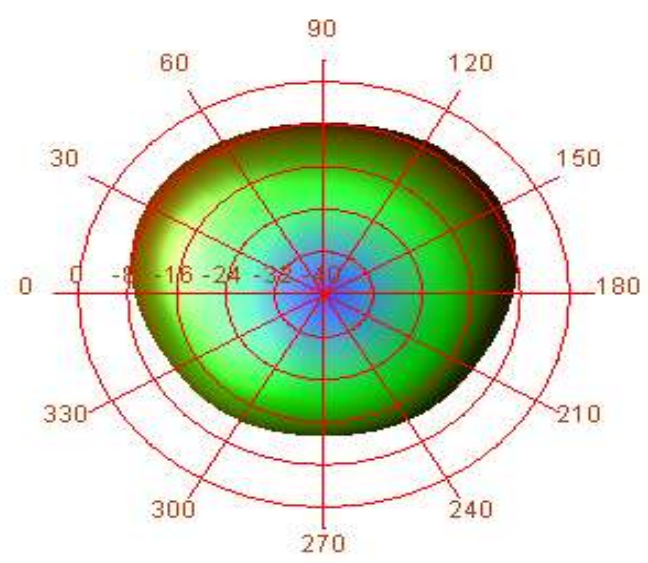

Fig. 8 Simulated result for radiation pattern 


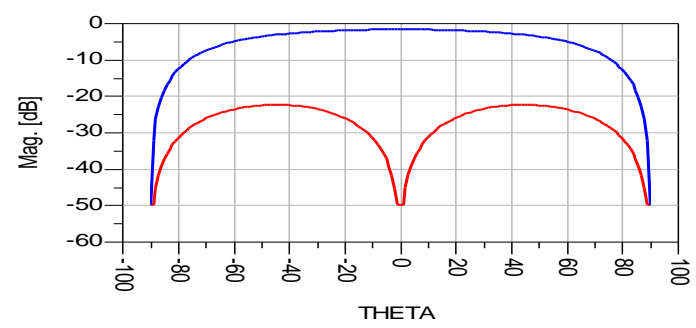

Fig.9 Simulated results for linear polarization

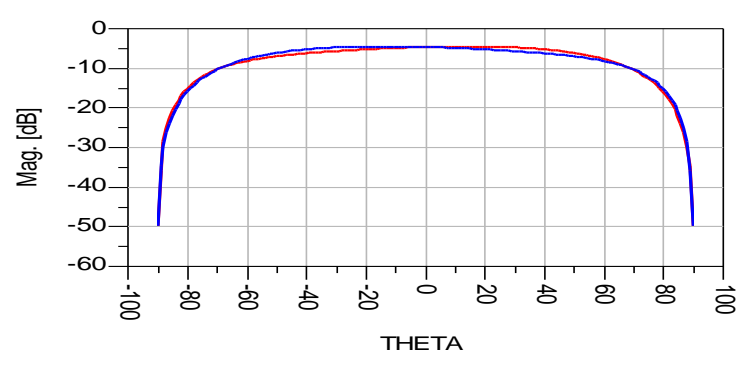

Fig.10 Simulated results for circular polarization

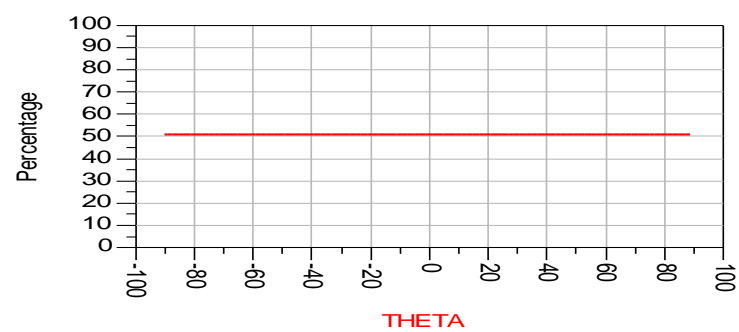

Fig.11 Simulated results for efficiency

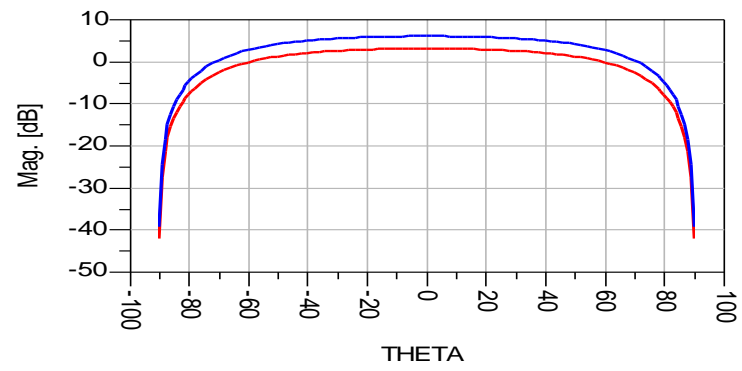

Fig.12 Simulated results for directivity and gain

TABLE I

Simulated antenna parameters

\begin{tabular}{|c|c|c|c|c|}
\hline Frequency(GHz) & $\begin{array}{l}\text { Power radiated } \\
\text { (watts) }\end{array}$ & $\begin{array}{l}\text { Directivity } \\
(\mathbf{d B})\end{array}$ & $\begin{array}{l}\text { Gain } \\
(\mathbf{d B})\end{array}$ & $\begin{array}{l}\text { Efficiency } \\
(\%)\end{array}$ \\
\hline 40 & 0.0109 & 7.7 & 4.7 & 50 \\
\hline
\end{tabular}




\section{Conclusion}

The objective was achieved by designing a conventional irregular octagonal microstrip patch antenna for cognitive radio applications. This is an ultra wide band (UWB) antenna having reflection loss less than $-20 \mathrm{~dB}$ for $40 \mathrm{GHz}$. The projections from octagonal antenna are responsible for wide band width and low reflection loss. The proposed design antenna incorporates advantages of both the designs and is a good design trade off showing better performances. This octagon loop structured antenna fed with microstrip line is designed to operate at $40 \mathrm{GHz}$ with circular polarization. The results confirm good performance of ultra wide band antenna design.

\section{References}

[1] Y. Tawk, C. G. Christodoulou , "A New reconfigurable antenna design for cognitive radio", IEEE , May,2009.

[2] T.Wu,E.L.Li,S.Y.Eom,K.lim,S,I.Jeon,J.Laskar,and M.M.Tentzeris"A multiband/scalable reconfigurable antenna for cognitive radio base stations",. IEEE, Trans, 2008.

[3] Y. Zhang, W. Hong, C. Yu J.-Y. Zhou Z.-Q. KuaiW. Tuttle bee, "Design and implementation of planar, ultra-wideband antennas with multiple notched bands based on stepped impedance resonators, IET Microw. Antennas Propag., 2009, Vol. 3, Iss. 7, pp. 1051-1059.

[4] P.H.Rao,V.F.Fusco and R. Cahill, “ Wideband Linear and Circularly Polarized Patch Antenna using a Printed Stepped T-Feed,” IEEE Trans. Antennas Propag. Vol. AP- 50, pp 356-361, March 2002.

[5] Hur, Y.; Park, J.; Woo, W.; Lim, K.; Lee, C.-H.; Kim, H.S.; Laskar, J., "A wideband analog multi-resolution spectrum sensing (MRSS) technique for cognitive radio (CR) systems," ISCAS 2006. Proceedings. IEEE International Symposium on Circuits and Systems May 2006, pp.21-24.

[6] Laskar, J.; Mukhopadhyay, R.; Hur, Y.; Lee, C.-H.; Lim, K.;Reconfigurable RFICs and modules for cognitiveradio'”, 2006 Topical Meeting on Silicon Monolithic Integrated Circuits in RF Systems, Jan. 2006. pp.18-20.

[7] Harada, Hiroshi; "A Software Defined Cognitive Radio Prototype" PIMRC 2007. IEEE 18th International Symposium on Personal, Indoor and Mobile Radio Communications, 2007,pp. 3-7.

[8] Patnam Hanumantha Rao :; "Antenna Configurations for Software Defined Radio and Cognitive Radio Communication Architecture" IEEE Trans, 2010

[9] Wen-jun Lu; Zhen-yuan Zhang; Ran Liu; Hong-bo Zhu "Design concept of a narrow wideband antenna for spectrum sensing applications” IEEE trans ,Microwave Conference Proceedings (CJMW), May 2011 ,PP 1 - 4.

[10] Al-Husseini, M.; Ramadan, A.; Zamudio, M.E.; Christodoulou, C.G.; El-Hajj, A.; Kabalan, K.Y. "A UWB antenna combined with a reconfigurable band pass filter for cognitive radio applications" Antennas and Propagation in Wireless communications (APWC), 2011 IEEE-APS Topical Conference Sep2011,PP 902 - 904.

[11] Hamid, M.R.; Gardner, P.; Hall, P.S.; Ghanem, F. "Review of reconfigurable vivaldi antennas" Antennas and Propagation Society International Symposium (APSURSI), 2010 IEEE July 2010, PP: 1 - 4.

[12] Kelly, J.R.; Ebrahimi, E.; Hall, P.S.; Gardner, P.; Ghanem, F. "Combined wideband and narrowband antennas for Cognitive Radio applications" Cognitive Radio and software Defined Radios: Technologies and Techniques, 2008 IET, Sep2008, PP: 1 - 4.

[13] Gunavathi, N.; Pandeeswari, R.; Raghavan, S. "A CPW-Fed Octagon-Shaped Aperture Antenna for Lower Band UWB Applications" India Conference (INDICON),Dec 2009 Annual IEEE conference.

[14] Choe, W.; Lee, J.K. “Analysis of higher order regular polygonal loop antennas" Antennas and Propagation, IEEE Transactions, Vol: 38, Issue: 7, July 1990, PP: $1114-1117$.

\section{Authors}

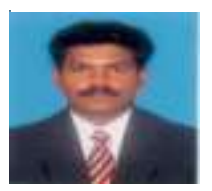

Mr. Siva sundara pandian.S is an Engineering Graduate in Electronics and Communication Engineering from Madurai Kamaraj University, (Tamilnadu), India. Did his Masters of Technology in VLSI design from Sathyabama University, Chennai (Tamilnadu). At present, he is pursuing PhD from Sathyabama University, Chennai (India). He has a total experience of 11 years which includes teaching as well as industrial. His research area is Microwave antennas and six port iunctions for cognitive radio.

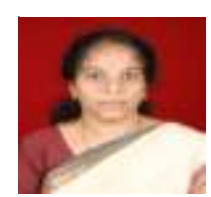

Dr.Suriyakala.C.D is an Engineering Graduate in Electronics and Communication Engineering from Manipal Institute of Technology, Manipal. Did her Masters M.S.(By Research) from Anna University University, Chennai (Tamilnadu) \& PhD form Sathyabama University. At present, She is associated with Electronics \& Tele communication Engg. Department, Sathyabama University, Chennai (Tamilnadu). She has a total experience of 19 years which includes teaching as well as industrial .Her research area is in software Agents for Communication. 